\title{
Sensitivity Analysis in Convex Optimization through the Circatangent Derivative
}

\author{
F. García - M. A. Melguizo Padial \\ Communicated by Marcin Studniarski
}

\begin{abstract}
The main goal of this paper is to analyse the sensitivity of a vector convex optimization problem according to variations in the right-hand side. We measure the quantitative behavior of a certain set of Pareto optimal points characterized to become minimum when the objective function is composed with a positive function. Its behavior is analysed quantitatively by using the circatangent derivative for set-valued maps. Particularly, it is shown that the sensitivity is closely related to a Lagrange multiplier solution of a dual program.
\end{abstract}

Keywords Convex optimization · Sensitivity Analysis · Set-valued map · Circatangent Derivative

Mathematics Subject Classification (2010) 90C25 · 90C29 · 54C60

F. García

Departamento de Matemática Aplicada, Universidad de Alicante, Carretera de San Vicente del Raspeig S/N, 03690 San Vicente del Raspeig, Alicante, Spain. E-mail: Fernando.gc@ua.es

\section{M.A. Melguizo Padial (Corresponding Author)}

Departamento de Matemática Aplicada, Universidad de Alicante, Carretera de San Vicente del Raspeig S/N, 03690 San Vicente del Raspeig, Alicante, Spain. E-mail: ma.mp@ua.es 


\section{Introduction}

This work deals with the problem of analysing the sensitivity of a vector convex optimization problem with respect to changes in the right-hand side. This topic has been widely studied by many authors since the works of Karush in 1939 and Kuhn and Tucker in 1951. Several significant results can be found in the papers [1-5].

There is a noteworthy fact that marks an essential difference between scalar and vectorial programming. Whereas in the case of scalar programming, the optimal point reached is a minimum point, and therefore unique; in the case of vectorial programming, the optimal ones are Pareto minimal or nondominated points. This fact implies that, in the vectorial case, the efficient set is not necessarily a singleton, and therefore, the problem of analysing its sensitivity becomes the problem of studying a point-to-set or set-valued map.

Traditionally, the problem generated by the appearance of this set-valued map has been solved by the implementation of selections in the efficient set (see, for example, $[6,7])$. In this way, the arisen set-valued map is reduced to a conventional point-to-point map, on which, subsequently, it is possible to study its differentiability. This approach has produced significant results. For example, in [5], using a selection of a special kind of optima, characterized to become minimum when the objective function is composed with a positive function $T$, the sensitivity of a convex vector optimization problem was measured. These optimal points are called $T$-optimal points and, under weak assumptions (see [8]), they are dense in the efficient set. This kind of optima has also been used in previous works such as [5-8], where it was analysed the sensitivity of different types of multiobjective problems.

An alternative to the use of selections is to introduce set-valued maps in the sensitivity analysis. This approach allows us to measure the evolution of certain sets of optima (not necessarily singletons) with respect to changes of some parameters (see, for example, the 
papers $[1,2])$. Following this methodology, the sensitivity of a differential vector optimization problem with equality constraints was analysed in [9]. Similarly, the sensitivity of a convex vector problem with inequality constraints was studied in [10]. In both cases, the study was carried out by analysing the derivability and by computing the contingent derivative of the set-valued map solution. In this paper, we deal with the same problem as in [10], but focusing on the tangential regularity of the set-valued solution map and the computation of its circatangent derivative, also called Clarke derivative.

The use of the circatangent derivative in sensitivity analysis assures some kind of "stability" in the obtained results. Intuitively, while the contingent cone of a set at a point consists in limits of directions that go from the set to the point, the Clarke tangent or circatangent cone "stabilizes" this tangency idea by allowing perturbations of the base point (see [11, page 138]). This fact makes particularly interesting the introduction of this derivative in the sensitivity analysis. The paper [12], by Chuong and Yao, is one of the first results in this direction. However, the price of this stability could sometimes be quite high since the circatangent cone may be too small or even reduced to the singleton $\{0\}$. This might occur for example in absence of convexity (see [13, page 129]). In those cases, the circatangent derivative does not provide any information, and the sensitivity analysis must be accomplished by using others set-valued derivatives (contingent, paratingent, and so on).

The article is organized as follows. Section 2 introduces notation, basic concepts, and some results that will be used throughout the paper. Section 3 is devoted to identify some regularity conditions that allow us to extend some useful properties of Fréchet differentiable maps to tangentially regular set-valued maps. Theorem 3.1 constitutes the main result of this section. Sensitivity analysis is the aim of Section 4. Namely, Theorem 4.2 states that the sensitivity of the problem, measured through the circatangent derivative, depends on a suitable Lagrange multiplier, solution of a dual problem, and its derivative. 


\section{Notation and Preliminaries}

Let $X, Y, Z$ and $W$ be four Banach spaces and let assume that $Y, Z$ and $W$ are ordered vector spaces with positive cones $Y_{+}, Z_{+}$and $W_{+}$, respectively, being the orders of $Y$ and $W$ antisymmetric and the order of $W$ verifying the infimum axiom (i.e., every non-empty order-bounded from below subset of $W$ has an infimum in $W$ ). From now on, the cones $Y_{+}$, $Z_{+}$, and $W_{+}$are assumed to be closed and $Z_{+}$and $W_{+}$with non-empty interior.

Let $T: Y \rightarrow W$ be a positive (i.e., $\left.T\left(Y_{+} \backslash\{0\}\right) \subset W_{+} \backslash\{0\}\right)$, linear, and continuous surjective map such that $\operatorname{Ker} T$ has a topological supplement, $Y_{T}$. Let $\widehat{T}$ denote the restriction of $T$ to $Y_{T}$ and $\pi$ the natural projection from $Y$ onto $\operatorname{Ker} T$. It follows from the open mapping Theorem (Theorem 2.11 in [14]) that the inverse operator $\widehat{T}^{-1}$ is continuous. Let denote by $V \subset Z$ an open and convex set such that $0 \notin V$, by $D \subset X$ a convex set, and by $f: D \rightarrow Y$ and $g: D \rightarrow Z$ two convex functions. Consider now the problem

$$
\operatorname{Min} f(x) \text { s.t. } x \in D, g(x) \leq b \text {, }
$$

for every $b \in V$.

It is said that a feasible point $x_{b}$ of Problem (1) (i.e., $x_{b} \in D$ and $g\left(x_{b}\right) \leq b$ ) is a $T$-optimal solution of Problem (1) iff $T f\left(x_{b}\right) \leq T f(x)$ for every $x \in D$ such that $g(x) \leq b$. If $x_{b}$ is a $T$-optimal solution of Problem (1), then $f\left(x_{b}\right)$ is an optimal point of Problem (1), i. e., $f\left(x_{b}\right)-f(x) \notin Y_{+} \backslash\{0\}$ for every $x \in D$ such that $g(x) \leq b$.

By $\mathfrak{L}(Z, W)$ we will denote the space of all linear and continuous maps from $Z$ into $W$ endowed with the usual norm. We say that $L \in \mathfrak{L}(Z, W)$ is a Lagrange $T$-multiplier of Problem (1) iff $L \geq 0$ (i.e., $\left.L\left(Z_{+}\right) \subset W_{+}\right)$and

$$
\operatorname{Inf}\{T f(x): x \in D, g(x) \leq b\}=\operatorname{Inf}\{T f(x)+L(g(x)-b): x \in D\} \in W .
$$


Theorem 3 in [5] yields that, if there exists a $T$-optimal solution of Problem (1) and the Slater condition is satisfied (i.e., if there is $x_{1} \in D$ such that $g\left(x_{1}\right) \in-$ int $\left.Z_{+}\right)$, then there exists a Lagrange $T$-multiplier of Problem (1).

Let us define $\Gamma_{T}$ as the set of all the positive operators $L \in \mathfrak{L}(Z, W)$ such that the set

$$
\{T f(x)+L g(x): x \in D\}
$$

is an order-bounded from below subset of $W$. Let us set, for each $L \in \Gamma_{T}$,

$$
\varphi(T, L):=\operatorname{Inf}\{T f(x)+L g(x): x \in D\} \in W,
$$

and, for each $G \in \mathfrak{L}(Z, Y)$ such that $T G \in \Gamma_{T}$, the dual map

$$
\psi(T, G):=\widehat{T}^{-1} \varphi(T, T G) \in Y_{T}
$$

Now, let us consider the following dual program

$$
\operatorname{Max}(\psi(T, G)-G(b)) \text { s.t. } G \in \mathfrak{L}(Z, Y), T G \geq 0 \text {, and } T G \in \Gamma_{T}
$$

for every $b \in V$.

It is said that a feasible solution $G_{b} \in \mathfrak{L}(Z, Y)$ of Problem (2) is a $T$-optimal dual solution of Problem (2) iff

$$
T(\psi(T, G)-G(b)) \leq T\left(\psi\left(T, G_{b}\right)-G_{b}(b)\right),
$$

for every feasible solution $G \in \mathfrak{L}(Z, Y)$.

Finally, iff $x_{b}$ is a $T$-optimal solution of Problem (1), $G_{x_{b}}$ is a $T$-optimal dual solution of Problem (2), and

$$
f\left(x_{b}\right)=\psi\left(T, G_{x_{b}}\right)-G_{x_{b}}(b),
$$

then we say that $\left[x_{b}, G_{x_{b}}\right]$ are $T$-optimal associated solutions. Theorem 10 in [5] states that, given $x_{b}$ a $T$-optimal solution of Problem (1), there exists a $T$-optimal dual solution $G_{x_{b}}$ 
of Problem (2) such that $\left[x_{b}, G_{x_{b}}\right]$ are $T$-optimal associated solutions if, and only if, there exists a Lagrange $T$-multiplier $L_{b} \in \mathfrak{L}(Z, W)$ of Problem (1); in this case $L_{b}=T G_{x_{b}}$.

Here, we recall some of the essential concepts of set-valued analysis that we will use throughout this work (further details can be found in [13] or [11]).

Let $S$ be a normed space, $A \subset S$ a non-empty set, $\bar{A}$ its closure in the norm topology, and $x \in \bar{A}$. The Bouligand or contingent cone $T_{A}(x)$ is defined by

$$
T_{A}(x):=\left\{v \in S: \liminf _{h \rightarrow 0+} \frac{d(A, x+h v)}{h}=0\right\}
$$

Therefore, $v \in T_{A}(x)$ if, and only if, there exist two sequences, $\left\{h_{n}\right\}_{n=1}^{\infty} \subset \mathbb{R}_{+} \backslash\{0\}$ converging to 0 and $\left\{v_{n}\right\}_{n=1}^{\infty} \subset S$ converging to $v$, such that $x+h_{n} v_{n} \in A$ for every $n \in \mathbb{N}$. The intermediate or adjacent cone $T_{A}^{b}(x)$ is defined by

$$
T_{A}^{b}(x):=\left\{v \in S: \lim _{h \rightarrow 0+} \frac{d(A, x+h v)}{h}=0\right\} .
$$

Therefore, $v \in T_{A}^{b}(x)$ if, and only if, for every sequence $\left\{h_{n}\right\}_{n=1}^{\infty} \subset \mathbb{R}_{+} \backslash\{0\}$ converging to 0 , there exists a sequence $\left\{v_{n}\right\}_{n=1}^{\infty} \subset S$ converging to $v$ such that $x+h_{n} v_{n} \in A$ for every $n \in \mathbb{N}$. The Clarke or circatangent cone $C_{A}(x)$ is defined by

$$
C_{A}(x):=\left\{v \in S: \lim _{\substack{h \rightarrow 0^{+} \\ A \ni \hat{x} \rightarrow x}} \frac{d(A, \hat{x}+h v)}{h}=0\right\} .
$$

Therefore, $v \in C_{A}(x)$ if, and only if, for every two sequences, $\left\{h_{n}\right\}_{n=1}^{\infty} \subset \mathbb{R}_{+} \backslash\{0\}$ converging to 0 and $\left\{x_{n}\right\}_{n=1}^{\infty} \subset A$ converging to $x$, there exists a sequence $\left\{v_{n}\right\}_{n=1}^{\infty} \subset S$ converging to $v$ such that $x_{n}+h_{n} v_{n} \in A$ for every $n \in \mathbb{N}$. The following inclusions are fulfilled: $C_{A}(x) \subset T_{A}^{b}(x) \subset T_{A}(x)$.

Let us fix two normed spaces $S_{1}$ and $S_{2}$, a subset $A \subset S_{1}$, a set-valued map $F: A \subset S_{1} \rightrightarrows S_{2}$, and $x_{0} \in$ Dom $F:=\{x \in A: F(x) \neq \emptyset\} . F$ is said to be lower semicontinuous at $x_{0}$ iff, for every $y \in F\left(x_{0}\right)$ and any sequence $\left\{x_{n}\right\}_{n=1}^{\infty} \subset \operatorname{Dom}(F)$ converging to $x_{0}$, there exists a sequence $\left\{y_{n}\right\}_{n=1}^{\infty} \subset Y$ converging to $y$ such that $y_{n} \in F\left(x_{n}\right)$ 
for every $n \in \mathbb{N}$. Let $(x, y) \in$ Graph $F:=\left\{(x, y) \in S_{1} \times S_{2}: y \in F(x)\right\}$. The Bouligand or contingent derivative $D F(x, y)$ of $F$ at $(x, y)$ is the set-valued map from $S_{1}$ to $S_{2}$ defined by

$$
\operatorname{Graph} D F(x, y):=T_{\mathrm{Graph} F}(x, y),
$$

the adjacent derivative $D^{b} F(x, y)$ of $F$ at $(x, y)$ is the set-valued map from $S_{1}$ to $S_{2}$ defined by

$$
\operatorname{Graph} D^{b} F(x, y):=T_{\text {Graph } F}^{b}(x, y) \text {, }
$$

and the circatangent derivative is the set-valued map from $S_{1}$ to $S_{2}$ defined by

$$
\operatorname{Graph} C F(x, y):=C_{\mathrm{Graph} F}(x, y) \text {. }
$$

$F$ is said to be derivable at $(x, y) \in \operatorname{Graph} F$ iff $D F(x, y)=D^{b} F(x, y)$. If $F$ is singlevalued and Fréchet differentiable at $x$, then $F$ is also derivable at $(x, F(x))$ and we have $D F(x, y)(u)=F^{\prime}(x, u)$ for every $u \in S_{1} . F$ is said to be tangentially regular at the point $(x, y) \in$ Graph $F$ iff $D F(x, y)=C F(x, y)$. If $F$ is single-valued and continuously Fréchet differentiable at $x$, then $F$ is tangentially regular at $(x, F(x))$ and $C F(x, y)(u)=F^{\prime}(x, u)$ for every $u \in S_{1}$.

\section{Regularity Conditions for Tangentially Regular Set-Valued Maps}

This section is devoted to deduce some regularity conditions, that allow to extend a useful property of Fréchet differentiable maps to tangentially regular set-valued maps.

Let us consider throughout this section a set-valued map $\Sigma: V \subset Z \rightrightarrows \mathfrak{L}(Z, Y)$, a point $\left(b_{0}, G_{0}\right) \in$ Graph $\Sigma$, and the set-valued map $\check{\Sigma}: V \subset Z \rightrightarrows Y$ defined by $\check{\Sigma}(b):=\Sigma(b)(b)$ for every $b \in V$. 
If $\Sigma$ is a single-valued and Fréchet differentiable map, then $\check{\Sigma}$ is also Fréchet differentiable (see Lemma 11 in [5]). Nevertheless, this does not remain true for tangentially regular maps, even if they are derivable. In Theorem 6 of [9] it is proved that, when $\Sigma$ is derivable, a necessary and sufficient condition to guarantee the derivability of $\check{\Sigma}$ is that $\Sigma$ fulfils property $\mathcal{R}$ formulated in Definition 5 of [9]. Here we recall it.

Definition 3.1 The set-valued map $\Sigma$ satisfies property $\mathcal{R}$ at $\left(b_{0}, G_{0}\right)$ when, given three sequences $\left\{b_{n}\right\}_{n=1}^{\infty} \subset Z,\left\{h_{n}\right\}_{n=1}^{\infty} \subset \mathbb{R}_{+} \backslash\{0\}$, and $\left\{G_{n}\right\}_{n=1}^{\infty} \subset \mathfrak{L}(Z, Y)$ such that

a.1) $\left\{b_{n}\right\}_{n=1}^{\infty}$ is convergent and $\left\{h_{n}\right\}_{n=1}^{\infty}$ converges to 0 ,

a.2) $G_{n} \in \Sigma\left(b_{0}+h_{n} b_{n}\right)$ for every $n \in \mathbb{N}$ and the sequence

$$
\left\{\frac{G_{n}\left(b_{0}+h_{n} b_{n}\right)-G_{0}\left(b_{0}\right)}{h_{n}}\right\}_{n=1}^{\infty} \text { is convergent, }
$$

there exist two sequences, $\left\{\bar{b}_{n}\right\}_{n=1}^{\infty} \subset Z$ and $\left\{\bar{G}_{n}\right\}_{n=1}^{\infty} \subset \mathfrak{L}(Z, Y)$ such that

b.1) $\lim _{n \rightarrow \infty} \bar{b}_{n}=\lim _{n \rightarrow \infty} b_{n}$,

b.2) $\bar{G}_{n} \in \Sigma\left(b_{0}+h_{n} \bar{b}_{n}\right)$ for every $n \in \mathbb{N}$ and

$$
\lim _{n \rightarrow \infty} \frac{\bar{G}_{n}\left(b_{0}+h_{n} \bar{b}_{n}\right)-G_{0}\left(b_{0}\right)}{h_{n}}=\lim _{n \rightarrow \infty} \frac{G_{n}\left(b_{0}+h_{n} b_{n}\right)-G_{0}\left(b_{0}\right)}{h_{n}}
$$

b.3) the sequence

$$
\left\{\frac{\bar{G}_{n}-G_{0}}{h_{n}}\right\}_{n=1}^{\infty} \text { is convergent in } \mathfrak{L}(Z, Y) .
$$

Nonetheless, as the following example shows, property $\mathcal{R}$ is not sufficient to assure the tangential regularity of $\check{\Sigma}$ even though $\Sigma$ is tangentially regular. By $\mathbb{R}_{+}$we will denote the set of non-negative real numbers, by $\mathbf{0}_{\ell_{2}}$ the zero element of the Hilbert space $\ell_{2}$, and by $\mathbf{0}_{\mathfrak{L}}$, the zero element of $\mathfrak{L}\left(\mathbb{R}, \ell_{2}\right)$. 
Example 3.1 Let us define $\Sigma: \mathbb{R}_{+} \cup\left\{-\frac{1}{2^{i}}: i \in \mathbb{N}\right\} \rightrightarrows \mathfrak{L}\left(\mathbb{R}, \ell_{2}\right)$ by

$$
\Sigma(t)(x):=\left\{\begin{array}{l}
(t x, 0, \ldots, 0, \ldots), \quad \text { if } t \geq 0, \\
\left(0, \ldots, 0,2^{\frac{i}{2}} x, 0, \ldots\right), \quad \text { if } t=-\frac{1}{2^{i}} \text { with } i \in \mathbb{N},
\end{array}\right.
$$

for every $x \in \mathbb{R}$. Then $\Sigma$ satisfies property $\mathcal{R}$ and it is tangentially regular at $\left(0, \mathbf{0}_{\mathfrak{L}}\right)$, but $\check{\Sigma}$ is not tangentially regular at $\left(0, \mathbf{0}_{\ell_{2}}\right)$.

Proof It can easily be verified that $\Sigma$ satisfies property $\mathcal{R}$ at $\left(0, \mathbf{0}_{\mathfrak{L}}\right)$ and

$T_{\text {Graph } \Sigma}\left(0, \mathbf{0}_{\mathfrak{L}}\right)=C_{\text {Graph } \Sigma}\left(0, \mathbf{0}_{\mathfrak{L}}\right)=\left\{(t,(t x, 0, \ldots, 0, \ldots)) \in \mathbb{R}_{+} \times \mathfrak{L}\left(\mathbb{R}, \ell_{2}\right), x \in \mathbb{R}\right\}$

Since $\check{\Sigma}: \mathbb{R}_{+} \cup\left\{-\frac{1}{2^{i}}: i \in \mathbb{N}\right\} \rightrightarrows \ell_{2}$ is defined as

$$
\check{\Sigma}(t):=\Sigma(t)(t)=\left\{\begin{array}{l}
\left(t^{2}, 0, \ldots, 0, \ldots\right), \text { if } t \geq 0 \\
\overbrace{-\frac{i}{2}}^{\text {i-th coordinate }}, 0, \ldots), \text { if } t=-\frac{1}{2^{i}} \text { with } i \in \mathbb{N},
\end{array}\right.
$$

then

$$
T_{\text {Graph } \check{\Sigma}}\left(0, \mathbf{0}_{\ell_{2}}\right)=\left\{\left(t, \mathbf{0}_{\ell_{2}}\right) \in \mathbb{R}_{+} \times \ell_{2}\right\}
$$

while $C_{\text {Graph } \check{\Sigma}}\left(0, \mathbf{0}_{\ell_{2}}\right)=\left\{\left(0, \mathbf{0}_{\ell_{2}}\right)\right\}$. Thus, $\Sigma$ is tangentially regular while $\check{\Sigma}$ is not.

Consequently, we need to introduce other regularity condition to guarantee the tangential regularity of $\check{\Sigma}$.

Definition 3.2 The set-valued map $\Sigma$ satisfies property $\mathcal{S}$ at $\left(b_{0}, G_{0}\right)$ when, given two sequences $\left\{a_{n}\right\}_{n=1}^{\infty} \subset V$ and $\left\{R_{n}\right\}_{n=1}^{\infty} \subset \mathfrak{L}(Z, Y)$ such that

c.1) $\left\{a_{n}\right\}_{n=1}^{\infty}$ converges to $b_{0}$ and $R_{n} \in \Sigma\left(a_{n}\right)$ for every $n \in \mathbb{N}$,

c.2) $\left\{R_{n}\left(a_{n}\right)\right\}_{n=1}^{\infty}$ converges to $G_{0}\left(b_{0}\right)$, 
there exists a sequence $\left\{\bar{R}_{n}\right\}_{n=1}^{\infty} \subset \mathfrak{L}(Z, Y)$ such that

d.1) $\bar{R}_{n} \in \Sigma\left(a_{n}\right)$ and $\bar{R}_{n}\left(a_{n}\right)=R_{n}\left(a_{n}\right)$ for every $n \in \mathbb{N}$,

d.2) $\left\{\bar{R}_{n}\right\}_{n=1}^{\infty}$ converges to $G_{0}$.

Theorem 3.1 Suppose that the set-valued map $\Sigma$ is tangentially regular and satisfies properties $\mathcal{R}$ and $\mathcal{S}$ at $\left(b_{0}, G_{0}\right)$. Then the corresponding set-valued map $\check{\Sigma}$ is tangentially regular at $\left(b_{0}, G_{0}\left(b_{0}\right)\right)$ and

$$
C \check{\Sigma}\left(b_{0}, G_{0}\left(b_{0}\right)\right)(u)=C \Sigma\left(b_{0}, G_{0}\right)(u)\left(b_{0}\right)+G_{0}(u)
$$

for every $u \in Z$.

Proof Let $(u, v) \in T_{\text {Graph } \check{\Sigma}}\left(b_{0}, G_{0}\left(b_{0}\right)\right)$. Then there exist two sequences, $\left\{h_{n}\right\}_{n=1}^{\infty} \subset \mathbb{R}_{+} \backslash\{0\}$ converging to 0 and $\left\{\left(b_{n}, v_{n}\right)\right\}_{n=1}^{\infty} \subset Z \times Y$ converging to $(u, v)$ such that

$$
\left(b_{0}, G_{0}\left(b_{0}\right)\right)+h_{n}\left(b_{n}, v_{n}\right) \in \operatorname{Graph} \check{\Sigma}
$$

for every $n \in \mathbb{N}$. Therefore, for each $n \in \mathbb{N}$, there exists $G_{n} \in \Sigma\left(b_{0}+h_{n} b_{n}\right)$ such that

$$
v_{n}=\frac{G_{n}\left(b_{0}+h_{n} b_{n}\right)-G_{0}\left(b_{0}\right)}{h_{n}} .
$$

Since $\Sigma$ satisfies property $\mathcal{R}$, there exist two sequences, $\left\{\bar{b}_{n}\right\}_{n=1}^{\infty} \subset Z$ and $\left\{\bar{G}_{n}\right\}_{n=1}^{\infty} \subset \mathfrak{L}(Z, Y)$, such that $\left\{\bar{b}_{n}\right\}_{n=1}^{\infty}$ converges to $u, \bar{G}_{n} \in \Sigma\left(b_{0}+h_{n} \bar{b}_{n}\right)$ for every $n \in \mathbb{N}$, the following equality holds

$$
\lim _{n \rightarrow \infty} \frac{\bar{G}_{n}\left(b_{0}+h_{n} \bar{b}_{n}\right)-G_{0}\left(b_{0}\right)}{h_{n}}=v,
$$

and the sequence

$$
\left\{\frac{\bar{G}_{n}-G_{0}}{h_{n}}\right\}_{n=1}^{\infty}
$$


is convergent in $\mathfrak{L}(Z, Y)$. Setting

$$
G_{v}:=\lim _{n \rightarrow \infty} \frac{\bar{G}_{n}-G_{0}}{h_{n}} \in \mathfrak{L}(Z, Y)
$$

we obtain that $\left(u, G_{v}\right) \in T_{\text {Graph } \Sigma}\left(b_{0}, G_{0}\right)$, and thus, since $\Sigma$ is tangentially regular at $\left(b_{0}, G_{0}\right)$,

$$
\left(u, G_{v}\right) \in C_{\text {Graph } \Sigma}\left(b_{0}, G_{0}\right) .
$$

Let $\left\{\bar{h}_{n}\right\}_{n=1}^{\infty} \subset \mathbb{R}_{+} \backslash\{0\},\left\{a_{n}\right\}_{n=1}^{\infty} \subset V$, and $\left\{R_{n}\right\}_{n=1}^{\infty} \subset \mathfrak{L}(Z, Y)$ be three sequences such that $\left\{\bar{h}_{n}\right\}_{n=1}^{\infty}$ converges to $0,\left\{a_{n}\right\}_{n=1}^{\infty}$ converges to $b_{0}, R_{n} \in \Sigma\left(a_{n}\right)$ for every $n \in \mathbb{N}$, and $\left\{R_{n}\left(a_{n}\right)\right\}_{n=1}^{\infty}$ converges to $G_{0}\left(b_{0}\right)$.

Since $\Sigma$ satisfies property $\mathcal{S}$, there exists a sequence $\left\{\bar{R}_{n}\right\}_{n=1}^{\infty} \subset \mathfrak{L}(Z, Y)$ converging to $G_{0}$ such that $\bar{R}_{n} \in \Sigma\left(a_{n}\right)$ and $\bar{R}_{n}\left(a_{n}\right)=R_{n}\left(a_{n}\right)$ for every $n \in \mathbb{N}$. Therefore, from (5), there exists a sequence $\left\{\left(d_{n}, L_{n}\right)\right\}_{n=1}^{\infty} \subset Z \times \mathfrak{L}(Z, Y)$ converging to $\left(u, G_{v}\right)$ such that

$$
\left(a_{n}, \bar{R}_{n}\right)+\bar{h}_{n}\left(d_{n}, L_{n}\right) \in \operatorname{Graph} \Sigma,
$$

for every $n \in \mathbb{N}$. Taking for each $n \in \mathbb{N} \quad S_{n}:=\bar{R}_{n}+\bar{h}_{n} L_{n}$, we obtain that $S_{n} \in \Sigma\left(a_{n}+\bar{h}_{n} d_{n}\right)$ for every $n \in \mathbb{N}$ and

$$
\lim _{n \rightarrow \infty} \frac{S_{n}-\bar{R}_{n}}{\bar{h}_{n}}=\lim _{n \rightarrow \infty} L_{n}=G_{v}
$$

Hence

$$
\begin{aligned}
\lim _{n \rightarrow \infty} \frac{S_{n}\left(a_{n}+\bar{h}_{n} d_{n}\right)-\bar{R}_{n}\left(a_{n}\right)}{\bar{h}_{n}} & =\lim _{n \rightarrow \infty} \frac{S_{n}-R_{n}}{\bar{h}_{n}}\left(a_{n}\right)+S_{n}\left(d_{n}\right) \\
& =G_{v}\left(b_{0}\right)+G_{0}(u)=v,
\end{aligned}
$$

and therefore, since for every $n \in \mathbb{N}$

$$
\left(a_{n}, R_{n}\left(a_{n}\right)\right)+\bar{h}_{n}\left(d_{n}, \frac{S_{n}\left(a_{n}+\bar{h}_{n} d_{n}\right)-\bar{R}_{n}\left(a_{n}\right)}{\bar{h}_{n}}\right) \in \operatorname{Graph} \check{\Sigma},
$$

we finally obtain that $(u, v) \in C_{\mathrm{Graph} \check{\Sigma}}\left(b_{0}, G_{0}\left(b_{0}\right)\right)$. 
Equality (4) immediately follows from Theorem 6 in [9], taking into account that every tangentially regular set-valued map is derivable and its circatangent and contingent derivatives coincide.

The following example shows that $\Sigma$ and $\check{\Sigma}$ may be tangentially regular, while $\Sigma$ does not satisfy property $\mathcal{S}$

Example 3.2 Let us consider $\Sigma: \mathbb{R}^{2} \rightrightarrows \mathfrak{L}\left(\mathbb{R}^{2}, \mathbb{R}\right)$ defined by

$$
\Sigma\left(t_{1}, t_{2}\right):=\left\{\begin{array}{l}
(0,0), \text { if }\left(t_{1}, t_{2}\right) \in \mathbb{R}^{2} \backslash \cup_{n=1}^{\infty}\left\{\left(\frac{1}{2^{n}}, \frac{1}{2^{n}}\right)\right\}, \\
(1,-1), \text { if }\left(t_{1}, t_{2}\right) \in \cup_{n=1}^{\infty}\left\{\left(\frac{1}{2^{n}}, \frac{1}{2^{n}}\right)\right\},
\end{array}\right.
$$

and $\left(b_{0}, G_{0}\right)=((0,0),(0,0)) \in$ Graph $\Sigma$. Then $\Sigma$ and $\check{\Sigma}$ are tangentially regular; nevertheless, $\Sigma$ does not satisfy property $\mathcal{S}$.

Proof Some easy computations show that

$$
\begin{aligned}
T_{\text {Graph } \Sigma}((0,0),(0,0)) & =C_{\text {Graph } \Sigma}((0,0),(0,0))= \\
& =\left\{\left(\left(t_{1}, t_{2}\right),(0,0)\right) \in \mathbb{R}^{2} \times \mathbb{R}^{2}:\left(t_{1}, t_{2}\right) \in \mathbb{R}^{2}\right\},
\end{aligned}
$$

and

$$
T_{\text {Graph } \check{\Sigma}}((0,0), 0)=C_{\text {Graph } \check{\Sigma}}((0,0), 0)=\left\{\left(\left(t_{1}, t_{2}\right), 0\right) \in \mathbb{R}^{2}:\left(t_{1}, t_{2}\right) \in \mathbb{R}^{2}\right\} .
$$

Therefore, $\Sigma$ and $\check{\Sigma}$ are tangentially regular. Nevertheless, $\Sigma$ does not satisfy property $\mathcal{S}$, since for $a_{n}=\left(\frac{1}{2^{n}}, \frac{1}{2^{n}}\right)$ and $R_{n}=\Sigma\left(\frac{1}{2^{n}}, \frac{1}{2^{n}}\right)=(1,-1)$ for every $n \in \mathbb{N}$, there is not any sequence $\left\{\bar{R}_{n}\right\}_{n=1}^{\infty}$ such that $\bar{R}_{n} \in \Sigma\left(a_{n}\right)$ and $\left\{\bar{R}_{n}\right\}_{n=1}^{\infty}$ converges to $(0,0)$. 


\section{Sensitivity Analysis}

From now on, let us consider the two following set-valued maps.

Definition 4.1 The $T$-optimal solution set-valued map of (1), $\Upsilon$, and the $T$-optimal dual solution set-valued map of Problem (1), $\Psi$, are defined by

$$
\begin{aligned}
\Upsilon: V & \rightrightarrows Y, \\
b & \rightrightarrows(b):=\left\{f\left(x_{b}\right): x_{b} \text { is a } T \text {-optimal solution of }(1)\right\}
\end{aligned}
$$

and

$$
\begin{aligned}
\Psi: V & \rightrightarrows Y, \\
b \rightrightarrows \Psi(b):=\left\{G_{x_{b}} \in \mathfrak{L}(Z, Y): x_{b} \text { is a } T \text {-optimal solution of }(1)\right. & \text { and } \left.\left[x_{b}, G_{x_{b}}\right] \text { are associated solutions }\right\},
\end{aligned}
$$

where $V \subset Z$ is an open and convex set such that $0 \notin V$.

Remark 4.1 If $\Psi$ is lower semicontinuous in $V$, from Lemma 2 of [10], it follows that $T \Psi$ is a single-valued map.

Theorem 4.1 Let $\left(b_{0}, G_{0}\right) \in$ Graph $\Psi$ and $\check{\Psi}: V \rightrightarrows Y$ be the set-valued map defined by $\check{\Psi}(b):=\Psi(b)(b)$ for every $b \in V$. If $\Psi$ is lower semicontinuous in $V$, tangentially regular at $\left(b_{0}, G_{0}\right)$, and $T \Psi$ is Fréchet differentiable at $b_{0} \in V$, then $\check{\Psi}$ is lower semicontinuous on $V$, tangentially regular at $\left(b_{0}, G_{0}\left(b_{0}\right)\right)$, and

$$
C \check{\Psi}\left(b_{0}, G_{0}\left(b_{0}\right)\right)(u)=C \Psi\left(b_{0}, G_{0}\right)(u)\left(b_{0}\right)+G_{0}(u)
$$

for every $u \in Z$.

Proof The lower semicontinuity of $\check{\Psi}$ easily follows from the direct application of the definition; therefore, the proof is omitted. Let us prove the tangential regularity of $\check{\Psi}$. Since $\Psi$ 
satisfies property $\mathcal{R}$ (see the proof of Theorem 4 in [10]), by using Theorem 3.1, we have just to prove that $\Psi$ satisfies property $\mathcal{S}$ at $\left(b_{0}, G_{0}\right)$.

Let $\left\{a_{n}\right\}_{n=1}^{\infty} \subset V$ and $\left\{R_{n}\right\}_{n=1}^{\infty} \subset \mathfrak{L}(Z, Y)$ be two sequences such that $\left\{a_{n}\right\}_{n=1}^{\infty}$ converges to $b_{0}, R_{n} \in \Psi\left(a_{n}\right)$ for every $n \in \mathbb{N}$, and $\left\{R_{n}\left(a_{n}\right)\right\}_{n=1}^{\infty}$ converges to $G_{0}\left(b_{0}\right)$.

Let $x_{a_{n}}$ be a $T$-optimal solution of (1) associated to $R_{n}$, and consider $J\left[R_{n}\right]$ the linear and continuous map defined as

$$
J\left[R_{n}\right](z):=\hat{T}^{-1} T R_{n}(z)-\pi f\left(x_{a_{n}}\right) \beta\left(a_{n}\right)(z),
$$

for every $z \in Z$ and $n \in \mathbb{N}$, where $\beta: V \rightarrow Z^{*}$ is a continuously Fréchet differentiable function in $V$ such that $\beta(b)(b)=1$ for every $b \in V^{1}$. By $Z^{*}$ we denote the topological dual space of $Z$.

Let us check that the map $J\left[R_{n}\right]$ is a $T$-optimal dual solution of (2) associated with $x_{a_{n}}$ for every $n \in \mathbb{N}$.

Indeed, since $R_{n}$ is a $T$-optimal dual solution of (2), from (7) we get that

$$
T J\left[R_{n}\right]=T R_{n} \geq 0
$$

for every $n \in \mathbb{N}$. Therefore, $\varphi\left(T, T R_{n}\right)=\varphi\left(T, T J\left[R_{n}\right]\right)$, and then,

$$
\begin{aligned}
\varphi\left(T, T J\left[R_{n}\right]\right) & =\varphi\left(T, T R_{n}\right) \\
& =\operatorname{Inf}\left\{T f(x)+T R_{n} g(x): x \in D\right\} \\
& =\operatorname{Inf}\left\{T f(x)+T R_{n}\left(g(x)-a_{n}\right): x \in D\right\}+T R_{n}\left(a_{n}\right)
\end{aligned}
$$

for every $n \in \mathbb{N}$. Thus,

$$
\varphi\left(T, T J\left[R_{n}\right]\right)=T f\left(x_{a_{n}}\right)+T R_{n}\left(a_{n}\right),
$$

\footnotetext{
1 The existence of such a $\beta$ comes from a separation theorem. Indeed, since $V$ is convex and $0 \notin V$, Theorem 3.4 in [14] provides a $z_{0}^{*} \in Z^{*}$ such that $z_{0}^{*}(0)<z_{0}^{*}(b)$ for every $b \in V$. The function $\beta: V \rightarrow Z^{*}$, defined as $\beta(b)=z_{0}^{*} / z_{0}^{*}(b)$, satisfies the required conditions.
} 
and hence,

$$
T J\left[R_{n}\right] \in \Gamma_{T}
$$

for every $n \in \mathbb{N}$. Consequently, from (8) and (10) we get that $J\left[R_{n}\right]$ is a dual feasible solution of (2) for every $n \in \mathbb{N}$.

On the other side, since $R_{n}$ is a $T$-optimal dual solution of (2),

$$
T\left(\psi(T, G)-G\left(a_{n}\right)\right) \leq T\left(\psi\left(T, R_{n}\right)-R_{n}\left(a_{n}\right)\right)
$$

for each dual feasible solution $G \in \mathfrak{L}(Z, Y)$ of (2), and then, from (8) it follows that

$$
\psi\left(T, R_{n}\right)=\hat{T}^{-1} \varphi\left(T, T R_{n}\right)=\hat{T}^{-1} \varphi\left(T, T J\left[R_{n}\right]\right)=\psi\left(T, J\left[R_{n}\right]\right)
$$

and $T J\left[R_{n}\right]\left(a_{n}\right)=T R_{n}\left(a_{n}\right)$ for every $n \in \mathbb{N}$. Therefore,

$$
T\left(\psi(T, G)-G\left(a_{n}\right)\right) \leq T\left(\psi\left(T, J\left[R_{n}\right]\right)-J\left[R_{n}\right]\left(a_{n}\right)\right)
$$

for each dual feasible solutions $G \in \mathfrak{L}(Z, Y)$ of (2), that is, $J\left[R_{n}\right]$ is $T$-optimal dual solution of (2) for every $n \in \mathbb{N}$.

Finally, from (9) it follows that

$$
\psi\left(T, J\left[R_{n}\right]\right)=\hat{T}^{-1} T f\left(x_{a_{n}}\right)+\hat{T}^{-1} T R_{n}\left(a_{n}\right)
$$

for every $n \in \mathbb{N}$. Now, since

$$
J\left[R_{n}\right]\left(a_{n}\right)=\hat{T}^{-1} T R_{n}\left(a_{n}\right)-\pi f\left(x_{a_{n}}\right) \beta\left(a_{n}\right)\left(a_{n}\right)=\hat{T}^{-1} T R_{n}\left(a_{n}\right)-\pi f\left(x_{a_{n}}\right),
$$

we get that

$$
\hat{T}^{-1} T R_{n}\left(a_{n}\right)=J\left[R_{n}\right]\left(a_{n}\right)+\pi f\left(x_{a_{n}}\right),
$$

and then, from (12), we obtain that

$$
\psi\left(T, J\left[R_{n}\right]\right)=\hat{T}^{-1} T f\left(x_{a_{n}}\right)+J\left[R_{n}\right]\left(a_{n}\right)+\pi f\left(x_{a_{n}}\right)=f\left(x_{a_{n}}\right)+J\left[R_{n}\right]\left(a_{n}\right),
$$


that is,

$$
f\left(x_{a_{n}}\right)=\psi\left(T, J\left[R_{n}\right]\right)-J\left[R_{n}\right]\left(a_{n}\right)
$$

for every $n \in \mathbb{N}$. Consequently, $J\left[R_{n}\right]$ is a $T$-optimal dual solution of (2) associated with $x_{a_{n}}$ for every $n \in \mathbb{N}$.

Likewise, if $x_{b_{0}}$ is the $T$-optimal solution of (1) associated with $G_{0}$, then the linear and continuous map $J\left[G_{0}\right]$, defined as

$$
J\left[G_{0}\right](z):=\hat{T}^{-1} T G_{0}(z)-\pi f\left(x_{b_{0}}\right) \beta\left(b_{0}\right)(z)
$$

for every $z \in Z$, is a $T$-optimal dual solution of (2) associated with $x_{b_{0}}$.

Consider now the sequence $\left\{\bar{R}_{n}\right\}_{n=1}^{\infty} \subset \mathfrak{L}(Z, Y)$, defined as

$$
\bar{R}_{n}(z):=J\left[R_{n}\right](z)+\left(G_{0}-J\left[G_{0}\right]\right)(z)-\left(G_{0}-J\left[G_{0}\right]\right)\left(a_{n}\right) \beta\left(a_{n}\right)(z),
$$

for every $z \in Z$ and $n \in \mathbb{N}$.

To complete the proof we will check that $\bar{R}_{n}\left(a_{n}\right)=R_{n}\left(a_{n}\right), \bar{R}_{n} \in \Psi\left(a_{n}\right)$ for every $n \in \mathbb{N}$, and that $\lim _{n \rightarrow \infty} \bar{R}_{n}=G_{0}$.

Indeed, since $\left[x_{a_{n}}, R_{n}\right]$ are associated solutions, from (3) we get that

$$
\pi f\left(x_{a_{n}}\right)=-\pi R_{n}\left(a_{n}\right)
$$

and thus,

$$
\begin{aligned}
\bar{R}_{n}\left(a_{n}\right) & =J\left[R_{n}\right]\left(a_{n}\right)=\hat{T}^{-1} T R_{n}\left(a_{n}\right)-\pi f\left(x_{a_{n}}\right)= \\
& =\hat{T}^{-1} T R_{n}\left(a_{n}\right)+\pi R_{n}\left(a_{n}\right)=R_{n}\left(a_{n}\right),
\end{aligned}
$$

for every $n \in \mathbb{N}$.

Moreover, since $T R_{n}=T J\left[R_{n}\right]$ and

$$
\left(G_{0}-J\left[G_{0}\right]\right)(z)=\left(G_{0}-\hat{T}^{-1} T G_{0}\right)(z)+\pi f\left(x_{b_{0}}\right) \beta\left(b_{0}\right)(z) \in \operatorname{Ker} T,
$$

for every $z \in Z$, we get that

$$
T \bar{R}_{n}=T R_{n}
$$


for every $n \in \mathbb{N}$. Now, from (3), (14) and (16) we obtain that $\left[x_{a_{n}}, \bar{R}_{n}\right]$ are

$T$-optimal associated solutions and, therefore, $\bar{R}_{n} \in \Psi\left(a_{n}\right)$ for every $n \in \mathbb{N}$.

Finally, let us check that

$$
\lim _{n \rightarrow \infty} \bar{R}_{n}=G_{0}
$$

Indeed, from (13) we have that

$$
\lim _{n \rightarrow \infty} \bar{R}_{n}=G_{0}-J\left[G_{0}\right]+\lim _{n \rightarrow \infty} J\left[R_{n}\right]-\lim _{n \rightarrow \infty}\left(G_{0}-J\left[G_{0}\right]\right)\left(a_{n}\right) \beta\left(a_{n}\right) .
$$

Let us consider now separately the two limits on the right-hand side of (17).

On one side we have that

$$
\lim _{n \rightarrow \infty} J\left[R_{n}\right]=\lim _{n \rightarrow \infty} \hat{T}^{-1} T R_{n}-\lim _{n \rightarrow \infty} \pi f\left(x_{a_{n}}\right) \beta\left(a_{n}\right)
$$

Since $T \Psi$ is Fréchet differentiable at $b_{0}$, and hence continue, the continuity of $\hat{T}^{-1}$ directly yields that

$$
\lim _{n \rightarrow \infty} \hat{T}^{-1} T R_{n}=\hat{T}^{-1} T G_{0}
$$

Furthermore, since $T R_{n}=T J\left[R_{n}\right]$, taking into account that both $R_{n}$ and $J\left[R_{n}\right]$ are associated solutions with $x_{a_{n}}$, from (3) we have that $R_{n}\left(a_{n}\right)=J\left[R_{n}\right]\left(a_{n}\right)$ for every $n \in \mathbb{N}$, and thus, from (14) we get that $\left\{J\left[R_{n}\right]\left(a_{n}\right)\right\}_{n=1}^{\infty}$ converges to $G_{0}\left(b_{0}\right)$. Likewise, since $T G_{0}=T J\left[G_{0}\right]$, from (3) it follows that $G_{0}\left(b_{0}\right)=J\left[G_{0}\right]\left(b_{0}\right)$. Hence $\left\{J\left[R_{n}\right]\left(a_{n}\right)\right\}_{n=1}^{\infty}$ converges to $J\left[G_{0}\right]\left(b_{0}\right)$, and therefore, since

$$
\begin{aligned}
& \left\|J\left[R_{n}\right]\left(a_{n}\right)-J\left[G_{0}\right]\left(b_{0}\right)\right\|= \\
& =\left\|\hat{T}^{-1} T R_{n}\left(a_{n}\right)-\pi f\left(x_{a_{n}}\right) \beta\left(a_{n}\right)\left(a_{n}\right)-\left(\hat{T}^{-1} T G_{0}\left(b_{0}\right)-\pi f\left(x_{b_{0}}\right) \beta\left(b_{0}\right)\left(b_{0}\right)\right)\right\| \geq \\
& \quad \geq\left|\left\|\hat{T}^{-1} T R_{n}\left(a_{n}\right)-\hat{T}^{-1} T G_{0}\left(b_{0}\right)\right\|-\left\|\pi f\left(x_{a_{n}}\right)-\pi f\left(x_{b_{0}}\right)\right\|\right|,
\end{aligned}
$$

and

$$
\lim _{n \rightarrow \infty}\left\|\hat{T}^{-1} T R_{n}\left(a_{n}\right)-\hat{T}^{-1} T G_{0}\left(b_{0}\right)\right\|=0
$$


we obtain that

$$
\lim _{n \rightarrow \infty} \pi f\left(x_{a_{n}}\right)=\pi f\left(x_{b_{0}}\right) .
$$

Thus

$$
\lim _{n \rightarrow \infty} \pi f\left(x_{a_{n}}\right) \beta\left(a_{n}\right)=\pi f\left(x_{b_{0}}\right) \beta\left(b_{0}\right),
$$

and hence, from (18) we have

$$
\lim _{n \rightarrow \infty} J\left[R_{n}\right]=\hat{T}^{-1} T G_{0}-\pi f\left(x_{b_{0}}\right) \beta\left(b_{0}\right)=J\left[G_{0}\right] .
$$

On the other side,

$$
\lim _{n \rightarrow \infty}\left(G_{0}-J\left[G_{0}\right]\right)\left(a_{n}\right) \beta\left(a_{n}\right)=\left(G_{0}-J\left[G_{0}\right]\right)\left(b_{0}\right) \beta\left(b_{0}\right)=0 .
$$

Therefore, from (17), we obtain that

$$
\lim _{n \rightarrow \infty} \bar{R}_{n}=G_{0}-J\left[G_{0}\right]+J\left[G_{0}\right]=G_{0}
$$

The following example shows that, in the former result, the assumption of tangential regularity on $\Psi$ can not be dropped. Hence, we can claim that the former theorem is a strengthening of Theorem 4 in [10].

Example 4.1 Let us define $\Sigma: \mathbb{R}_{+} \cup\left\{-\frac{1}{2^{i}}: i \in \mathbb{N}\right\} \rightrightarrows \mathfrak{L}\left(\mathbb{R}, \ell_{2}\right)$ by

$$
\Sigma(t)(x):=\left\{\begin{array}{l}
(t x, 0, \ldots, 0, \ldots), \quad \text { if } t \geq 0, \\
(0, \ldots, 0, \overbrace{\left.2^{-\frac{i}{2}} x, 0, \ldots\right),}^{\text {i-th coordinate }} \quad \text { if } t=-\frac{1}{2^{i}} \text { with } i \in \mathbb{N},
\end{array}\right.
$$

for every $x \in \mathbb{R}$. Then $\Sigma$ satisfies properties $\mathcal{R}$ and $\mathcal{S}$ at $\left(0, \mathbf{0}_{\mathfrak{L}}\right), \Sigma$ is not tangentially regular at $\left(0, \mathbf{0}_{\mathfrak{L}}\right)$, and $\check{\Sigma}$ is not tangentially regular at $\left(0, \mathbf{0}_{\ell_{2}}\right)$ either. 
Proof It can easily be verified that $\Sigma$ satisfies properties $\mathcal{R}$ and $\mathcal{S}$ at $\left(0, \mathbf{0}_{\mathfrak{L}}\right)$ and

$$
T_{\text {Graph } \Sigma}\left(0, \mathbf{0}_{\mathfrak{L}}\right)=\left\{\left(t, \mathbf{0}_{\mathfrak{L}}\right) \in \mathbb{R} \times \mathfrak{L}\left(\mathbb{R}, \ell_{2}\right): t \geq 0\right\}
$$

while $C_{\text {Graph } \check{\Sigma}}\left(0, \mathbf{0}_{\ell_{2}}\right)=\left\{\left(0, \mathbf{0}_{\ell_{2}}\right)\right\}$. Thus, $\Sigma$ is not tangentially regular at $\left(0, \mathbf{0}_{\mathfrak{L}}\right)$. Since $\check{\Sigma}: \mathbb{R}_{+} \cup\left\{-\frac{1}{2^{i}}: i \in \mathbb{N}\right\} \rightrightarrows \ell_{2}$ is defined by

$$
\check{\Sigma}(t):=\Sigma(t)(t)=\left\{\begin{array}{l}
\left(t^{2}, 0, \ldots, 0, \ldots\right), \text { if } t \geq 0, \\
(0, \ldots, 0,-\overbrace{-\frac{3}{2} i}^{\text {i-th }}, 0, \ldots), \text { if } t=-\frac{1}{2^{i}} \text { with } i \in \mathbb{N},
\end{array}\right.
$$

then

$$
T_{\text {Graph } \check{\Sigma}}\left(0, \mathbf{0}_{\ell_{2}}\right)=\left\{\left(t, \mathbf{0}_{\ell_{2}}\right) \in \mathbb{R} \times \ell_{2}: t \geq 0\right\},
$$

while $C_{\text {Graph } \check{\Sigma}}\left(0, \mathbf{0}_{\ell_{2}}\right)=\left\{\left(0, \mathbf{0}_{\ell_{2}}\right)\right\}$. Thus, $\check{\Sigma}$ is no tangentially regular at $\left(0, \mathbf{0}_{\ell_{2}}\right)$.

Theorem 4.2 Let $\left(b_{0}, f\left(x_{b_{0}}\right)\right) \in$ Graph $\Upsilon$. If $\Psi$ is lower semicontinuous on $V$, tangentially regular at $\left(b_{0}, G_{x_{b_{0}}}\right)$, and $T \Psi$ is continuously Fréchet differentiable at $b_{0}$, then $\Upsilon$ is lower semicontinuous at $b_{0}$, tangentially regular at $\left(b_{0}, f\left(x_{b_{0}}\right)\right)$, and

$$
C \Upsilon\left(b_{0}, f\left(x_{b_{0}}\right)\right)(u)=-G_{x_{b_{0}}}(u)-\pi C \Psi\left(b_{0}, G_{x_{b_{0}}}\right)(u)\left(b_{0}\right)
$$

for every $u \in Z$.

Proof Let $\check{\Psi}: V \rightrightarrows Y$ be the set-valued map defined as $\check{\Psi}(b):=\Psi(b)(b)$ for every $b \in V$.

Since

$$
\Upsilon(b)=\psi(T, \Psi(b))-\check{\Psi}(b),
$$

for every $b \in V$, we have

$$
T \Upsilon(b)=\varphi(T, T \Psi(b))-T \check{\Psi}(b)
$$


for every $b \in V$. The proof ${ }^{2}$ of Lemma 2 in [10] yields that $T \Upsilon$ is continuously Fréchet differentiable at $b_{0}$, and thus, by Theorem 5 and Theorem 10 in [5] we obtain that

$$
(T \Upsilon)^{\prime}\left(b_{0}, u\right)=-T \Psi\left(b_{0}\right)(u) \text {, for every } u \in Z \text {. }
$$

Now, from Lemma 11 of [5], we get that $T \check{\Psi}(b)$ is continuously Fréchet differentiable at $b_{0}$ and

$$
(T \check{\Psi})^{\prime}\left(b_{0}, u\right)=T \Psi\left(b_{0}\right)(u)+(T \Psi)^{\prime}\left(b_{0}, u\right)\left(b_{0}\right)
$$

for every $u \in Z$. Therefore, setting $\tilde{\varphi}(b):=\varphi(T, T \Psi(b))$ for every $b \in V$, from (21) it follows that

$$
-T \Psi\left(b_{0}\right)(u)=\tilde{\varphi}^{\prime}\left(b_{0}, u\right)-T \Psi\left(b_{0}\right)(u)-(T \Psi)^{\prime}\left(b_{0}, u\right)\left(b_{0}\right),
$$

for every $u \in Z$. Consequently, $\tilde{\varphi}$ is continuously Fréchet differentiable at $b_{0}$ and

$$
\tilde{\varphi}^{\prime}\left(b_{0}, u\right)=(T \Psi)^{\prime}\left(b_{0}, u\right)\left(b_{0}\right),
$$

for every $u \in Z$. Hence, taking $\tilde{\psi}(b):=\hat{T}^{-1} \tilde{\varphi}(b)=\psi(T, \Psi(b))$ for every $b \in V$, we get that $\tilde{\psi}$ is continuously Fréchet differentiable at $b_{0}$. Furthermore, since $\Psi$ is tangentially regular at $\left(b_{0}, G_{x_{b_{0}}}\right)$, from Theorem 4.1, we have that $\check{\Psi}$ is tangentially regular at $\left(b_{0}, G_{x_{b_{0}}}\left(b_{0}\right)\right)$. Therefore, applying Propositions 5.1.2 and 5.2.2 of [13] to (20), we obtain that $\Upsilon$ is tangentially regular at $\left(b_{0}, f\left(x_{b_{0}}\right)\right)$.

To finish the proof, let us note that (19) immediately follows from Theorem 6 in [10], since every tangentially regular set-valued map is derivable and its circatangent and contingent derivatives coincide.

The lower semicontinuity of $\Upsilon$ at $b_{0}$ directly follows from (20), taking into account the continuity of $\tilde{\varphi}$ at $b_{0}$ and Theorem 4.1 .

\footnotetext{
${ }^{2}$ The proof of Lemma 2 in [10] contains a misprint, the equality $[T \Upsilon]^{\prime}\left(b_{0}, u\right)=T G_{i}\left(b_{0}, u\right)$ must be replaced by $[T \Upsilon]^{\prime}\left(b_{0}, u\right)=-T G_{i}\left(b_{0}, u\right)$ for $i=1,2$.
} 
Remark 4.2 Note that the scalar case is included in Theorem 4.2 as a particular case. Indeed, taking $Z:=W:=\mathbb{R}$ and $T$ as the identity map, the set-valued map $\Upsilon$ becomes a conventional continuously Fréchet differentiable point-to-point map, and then, Theorem 4.2 takes the form

$$
\Upsilon^{\prime}\left(b_{0}, u\right)=C \Upsilon\left(b_{0}, f\left(x_{b_{0}}\right)\right)(u)=-G_{x_{b_{0}}}(u) \quad \forall u \in \mathbb{R}
$$

since $\operatorname{Ker} T=\{0\}$, and the circatangent derivative and the Fréchet differential coincide. Consequently, our approach extends the classical result from scalar to vector optimization by means of the circatangent derivative, providing a set-valued extension of this.

Definition 4.2 Let $p \in \mathbb{R} \backslash\{0\}$, it is said that (1) is $p$-homogeneous when the two following properties hold

i) $D$ is a convex cone,

ii) $f(t x)=t^{p} f(x)$ and $g(t x)=t^{p} g(x)$ for every $x \in D$ and $t>0$.

Note that linear problems are a very important particular case of 1-homogeneous problems. The following result is a particularization of Theorem 4.2 for $p$-homogeneous problems.

Corollary 4.1 Assume that Problem (1) is $p$-homogeneous for every $b \in V$ and the hypothesis of Theorem 4.2 hold. Then $\Upsilon$ is lower semicontinuous at $b_{0}$, tangentially regular at $\left(b_{0}, f\left(x_{b_{0}}\right)\right)$, and

$$
C \Upsilon\left(b_{0}, f\left(x_{b_{0}}\right)\right)(u)=-G_{x_{b_{0}}}(u)-C \Psi\left(b_{0}, G_{x_{b_{0}}}\right)(u)\left(b_{0}\right),
$$

for every $u \in Z$.

Proof From Theorem 3.2 of [15] it follows that 


$$
\Upsilon(b)=-\Psi(b)(b),
$$

for every $b \in V$. Thus, applying Theorem 4.1 to the previous equality, we get that $\Upsilon$ is lower semicontinuous at $b_{0}$, tangentially regular at $\left(b_{0}, f\left(x_{b_{0}}\right)\right)$, and

$$
C \Upsilon\left(b_{0}, f\left(x_{b_{0}}\right)\right)(u)=-G_{x_{b_{0}}}(u)-C \Psi\left(b_{0}, G_{x_{b_{0}}}\right)(u)\left(b_{0}\right)
$$

for every $u \in Z$.

Remark 4.3 Note that, under the assumptions of Corollary 4.1, we have that

$$
C \Psi\left(b_{0}, G_{x_{b_{0}}}\right)(u)\left(b_{0}\right)=\pi C \Psi\left(b_{0}, G_{x_{b_{0}}}\right)(u)\left(b_{0}\right),
$$

for every $u \in Z$. This is a direct consequence of (19) and (22).

The following example shows how Theorem 4.2 works.

Example 4.2 Let us define $\mu(\{n\}):=e^{-n} \forall n \in \mathbb{N}=\{0,1,2, \ldots\}$, and the Hilbert space

$$
L^{2}(\mu):=\left\{\left(\lambda_{n}\right)_{n} \in \mathbb{R}^{\mathbb{N}}: \sum_{n=0}^{+\infty} \lambda_{n}^{2} e^{-n}<+\infty\right\} .
$$

Let us consider $D:=X:=L^{2}(\mu), Y:=\mathbb{R}^{3}, Z:=W:=\mathbb{R}, V:=\left(\frac{9}{10}, \frac{11}{10}\right) \subset \mathbb{R}$, $T:=(1,1, \sqrt{2})$, and the problem

$$
\left\{\begin{array}{l}
\operatorname{Min}\left(-\sum_{n} u_{3 n+2} e^{-3 n-2}+\sum_{n} \sqrt{2} u_{3 n+1} e^{-3 n-1}-\sum_{n} u_{3 n} e^{-3 n}\right. \\
\quad-\sum_{n} u_{3 n+2} e^{-3 n-2}-\sum_{n} \sqrt{2} u_{3 n+1} e^{-3 n-1}-\sum_{n} u_{3 n} e^{-3 n} \\
\left.\quad \sum_{n} \sqrt{2} u_{3 n+2} e^{-3 n-2}-\sum_{n} \sqrt{2} u_{3 n} e^{-3 n}\right) ; \\
\cosh \left(\left[\sum_{n} u_{3 n} e^{-3 n}\right]^{2}\right)-\frac{1}{2} \leq b,\left(u_{n}\right)_{n} \in D .
\end{array}\right.
$$

Let us define $\phi(b):=\operatorname{argcosh}\left(b+\frac{1}{2}\right)$ for every $b \in V$. Solving Problem (23) we obtain the $T$-optimal solution set-valued map

$$
\Upsilon(b)=\{(-\sqrt{\phi(b)}+\sqrt{2} \mu-\lambda,-\sqrt{\phi(b)}-\sqrt{2} \mu-\lambda,-\sqrt{2 \phi(b)}+\sqrt{2} \lambda): \lambda, \mu \in \mathbb{R}\} .
$$


Let us study the sensitivity of (23) at $b=1, x_{1}=\left(\sqrt{\operatorname{argcosh} \frac{3}{2}}, 0,0, \cdots\right)$, and so $f\left(x_{1}\right)=\left(-\sqrt{\operatorname{argcosh} \frac{3}{2}},-\sqrt{\operatorname{argcosh} \frac{3}{2}},-\sqrt{2 \operatorname{argcosh} \frac{3}{2}}\right)$. For short, we will denote $a=\sqrt{\operatorname{argcosh} \frac{3}{2}}$. We first analyse the sensitivity by calculating

$$
\begin{aligned}
& C \Upsilon\left(1, f\left(x_{1}\right)\right)(u)= \\
& =\left\{\left(-\frac{\sqrt{5}}{5 a} u+\sqrt{2} \mu-\lambda,-\frac{\sqrt{5}}{5 a} u-\sqrt{2} \mu-\lambda,-\frac{\sqrt{10}}{5 a} u+\sqrt{2} \lambda\right): \lambda, \mu \in \mathbb{R}\right\} .
\end{aligned}
$$

Let us now apply Theorem 4.2 to verify (24). Since Ker $T$ is the linear space generated by $\{(-1,-1, \sqrt{2}),(1,-1,0)\}$, we have $G_{x_{1}}(u)=\left(\frac{\sqrt{5}}{5 a} u, \frac{\sqrt{5}}{5 a} u, \frac{\sqrt{10}}{5 a} u\right)$, and the $T$-optimal dual solution set-valued map of (23) is

$$
\Psi(b)=\left\{\left(\frac{2}{\xi(b)}+\frac{\sqrt{2} \mu-\lambda}{b}, \frac{2}{\xi(b)}-\frac{\sqrt{2} \mu+\lambda}{b}, \frac{2 \sqrt{2}}{\xi(b)}+\frac{\sqrt{2} \lambda}{b}\right): \lambda, \mu \in \mathbb{R}\right\},
$$

where $\xi(b):=\sqrt{\phi(b)} \sqrt{4 b-2} \sqrt{4 b+6}$ and $b \in V$. Thus, for every $u \in \mathbb{R}$, we have that

$$
\begin{aligned}
& C \Psi\left(1, G_{x_{1}}\right)(u)(1)=\{(\eta(a) u+\kappa(a) u-\sqrt{2} \mu+\lambda, \\
& \eta(a) u+\kappa(a) u+\sqrt{2} \mu+\lambda, \eta(a) u+\sqrt{2} \kappa(a) u-\sqrt{2} \lambda): \lambda, \mu \in \mathbb{R}\},
\end{aligned}
$$

where $\eta(a):=-\frac{1}{5 a^{3}}$ and $\kappa(a):=-\frac{6 \sqrt{5}}{25 a}$. Hence

$$
\pi C \Psi\left(1, G_{x_{1}}\right)(u)(1)=\{(-\sqrt{2} \mu+\lambda, \sqrt{2} \mu+\lambda,-\sqrt{2} \lambda): \lambda, \mu \in \mathbb{R}\} .
$$

Finally, we obtain that

$$
\begin{aligned}
& -G_{x_{1}}(u)-\pi C \Psi\left(1, G_{x_{1}}\right)(u)(1)= \\
& =\left\{-\left(\frac{\sqrt{5}}{5 a} u, \frac{\sqrt{5}}{5 a} u, \frac{\sqrt{10}}{5 a} u\right)-(-\sqrt{2} \mu+\lambda, \sqrt{2} \mu+\lambda,-\sqrt{2} \lambda): \lambda, \mu \in \mathbb{R}\right\}= \\
& =C \Upsilon\left(1, f\left(x_{1}\right)\right)(u),
\end{aligned}
$$

for every $u \in \mathbb{R}$, as Theorem 4.2 states.

Before ending this section we will particularize the obtained results for the case of a linear VOP in a finite-dimensional Euclidean space. For that, we will review some notation 
and terminology. Now, $f$ and $g$ are linear maps, $f: D \subset \mathbb{R}^{n} \rightarrow \mathbb{R}^{p}$ and $g: D \subset \mathbb{R}^{n} \rightarrow$ $\mathbb{R}^{m}$. Our problem is again,

$$
\operatorname{Min} f(x) \text { s.t. } x \in D, g(x) \leq b \text {, }
$$

for every $b \in V$. We fix $W=\mathbb{R}$, and consider $\lambda: \mathbb{R}^{p} \rightarrow \mathbb{R}$ a linear map, i.e., $\lambda=\left(\lambda_{i}\right) \in \mathbb{R}^{p}$, such that $\lambda_{i} \geq 0$ for all $i \in\{1, \cdots, p\}$ and $\sum_{i} \lambda_{i}=1$. Now let

$$
\Gamma_{\lambda}=\left\{\mu \in \mathbb{R}_{+}^{m}:\left\{\lambda^{T} f(x)+\mu^{T} g(x): x \in D\right\} \text { is bounded from below }\right\},
$$

$$
\varphi(\lambda, \mu)=\operatorname{Inf}\left\{\lambda^{T} f(x)+\mu^{T} g(x): x \in D\right\} \text { with } \mu \in \Gamma_{\lambda},
$$

and

$$
\psi(\lambda, \mu)=\frac{1}{\sum_{i} \lambda_{i}^{2}} \varphi(\lambda, \mu) \lambda
$$

Then, the dual problem has the form

$$
\operatorname{Max}\left(\psi\left(\lambda, G^{T} \lambda\right)-G b\right) \text { s. t. } G \in \mathbb{R}^{p \times m} \text { and } G^{T} \lambda \in \Gamma_{\lambda} \text {. }
$$

Now, Corollary 4.1 applies, and (22) is again obtained, i.e.,

$$
C \Upsilon\left(b_{0}, f\left(x_{b_{0}}\right)\right)(u)=-G_{x_{b_{0}}}(u)-C \Psi\left(b_{0}, G_{x_{b_{0}}}\right)(u)\left(b_{0}\right), \forall u \in \mathbb{R}^{m} .
$$

\section{Conclusions}

The objective of this article is to analyse the sensitivity of a convex multiobjective optimization program with inequality constraints. Fixed a positive, linear, and continuous map $T$, the $T$-solution set-valued map is defined as the correspondence that assigns to each value 
of the parameter (the right-hand side), the set of the Pareto minimal points of its associated program on which $T$ takes a minimum value. The behavior of the $T$-solution set-valued map is quantitatively analysed by using the circatangent derivative. The use of this derivative transmits to the obtained results its characteristic stability. The main result of the paper is Theorem 4.2, which states that the sensitivity of the program is measured by a Lagrange multiplier, solution of the dual program introduced in [5], plus the projection of its derivative onto the kernel of $T$. This theorem extends the classical result from scalar to vector programming by means of the circatangent derivative, providing a set-valued extension of this. As a particular case, a simpler version of Theorem 4.2 has been obtained for $p$-homogeneous problems. The study has been developed in the context of arbitrary Banach spaces. Therefore, the results are quite general and may be applied to many particular situations such as static, dynamic, or semi-infinite problems.

Finally, let us remark that Theorem 4.2 can be regarded as a first step in solving a more general problem in which the map $T$ is not considered fixed, but varies among all the possible linear, continuous and positive maps. In this case, under some reasonable conditions, the set of all the $T$-minimal points becomes dense in the efficient set, and thus, the study of its sensitivity turns out to be particularly interesting.

Acknowledgements The authors would like to express their very great appreciation to Professor Jiménez Guerra for his valuable suggestions during the planning and development of this research work. We would also like to extend our thanks to the anonymous referee and the editors for their tips and constructive critiques. Research partially supported by the University of Alicante, project GRE11-08.

\section{References}

1. Tanino T.: Sensitivity analysis in multiobjective optimization. J. Optim. Theory Appl. 56 479-499 (1988) 
2. Tanino T.: Stability and sensitivity analysis in convex vector optimization. SIAM J. Control Optim. Appl. $26521-536(1988)$

3. Klose J.: Sensitivity analysis using the tangent derivative. Numer. Funct. Anal. Optim. 13 143-153 (1992)

4. Kuk H., Tanino T., Tanaka M.: Sensitivity analysis in vector optimization. J. Optim. Theory Appl. 89 713-730 (1996)

5. Balbás A., Jiménez Guerra P.: Sensitivity analysis for convex multiobjective programming in abstract spaces. J. Math. Anal. Appl. 202 645-648 (1996)

6. Dauer J.P., Osman M.S.A.: Decomposition of the parametric space in multiobjective convex programs using the generalized Tchebycheff norm. J. Math. Anal. Appl. 107 156-166 (1985)

7. Hansen P., Labbé M., Wendell R.E.: Sensitivity analysis in multiple objective linear programming: The tolerance approach. European J. Oper. Res. 38 63-69 (1989)

8. Balbás A., Ballvé M., P. Jiménez Guerra M.: Density theorems for ideal points in vector optimization. European J. Oper. Res. 133 260-266 (2001)

9. Jiménez Guerra P., Melguizo M.A., Muñoz M.J.: Sensitivity analysis in multiobjective differential programming. Comput. Math. Appl. 52 109-120 (2006)

10. Jiménez Guerra P., Melguizo M.A., Muñoz M.J.: Sensitivity analysis in convex programming. Comput. Math. Appl. 58 1239-1246 (2009)

11. Borwein J.M., Lewis A.S.: Convex Analysis and Nonlinear Optimization. Theory and examples. Springer, Berlin, (2000)

12. Chuong T.D., Yao J.C.: Generalized Clarke epiderivatives of parametric vector optimization problems. J. Optim. Theory Appl. 146 77-94 (2010)

13. Aubin J. P., Frankowska H.: Set-Valued Analysis. Birkhäuser, Boston-Basel-Berlin, (1990)

14. Rudin W.: Functional Analysis. McGraw-Hill, New Delhi, (1977)

15. Balbás A., Ballvé M., Jiménez Guerra P.: Sensitivity in multiobjective programming under homogeneity assumptions. J. Multi- Crit. Decis. Anal. 8 133-138 (1999) 